Utah State University

DigitalCommons@USU

\title{
Genomic Variation of Introduced Salvinia minima in Southeastern United States
}

\author{
Carol A. Rowe \\ Utah State University \\ Donald P. Hauber \\ Loyola University
}

Paul G. Wolf

Utah State University

Follow this and additional works at: https://digitalcommons.usu.edu/eco_pubs

Part of the Ecology and Evolutionary Biology Commons

\section{Recommended Citation}

Rowe, C. A., Hauber, D. P., \& Wolf, P. G. (2018). Genomic variation of introduced Salvinia minima in southeastern United States. Aquatic Botany, 151, 38-42. https://doi.org/10.1016/j.aquabot.2018.07.011

This Article is brought to you for free and open access by the Ecology Center at DigitalCommons@USU. It has been accepted for inclusion in Ecology Center Publications by an authorized administrator of DigitalCommons@USU.

For more information, please contact

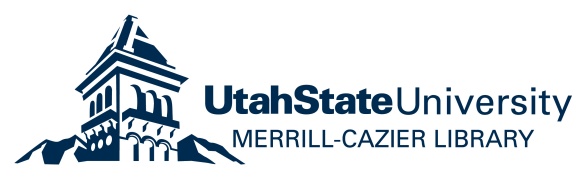


1 Genomic variation of introduced Salvinia minima in southeastern United States

3 Carol A. Rowe ${ }^{1}$, Donald P. Hauber ${ }^{2}$, Paul G. Wolf ${ }^{1,3,4}$

$4 \quad{ }^{1}$ Department of Biology, Utah State University, Logan, UT 84322, USA

$5 \quad{ }^{2}$ Department of Biological Sciences, Loyola University, New Orleans, LA 70118, USA

$6 \quad{ }^{3}$ Ecology Center, Utah State University, Logan, UT 84322, USA

$7 \quad{ }^{4}$ Author for correspondence (paul.wolf@usu.edu)

8

9 Declarations of interest:

10 None

11

12 Contributions of authors:

13 DPH and PGW conceived and planned the project

14 CAR and DPH performed the molecular work

15 CAR performed the data processing, exploration, and analysis

16 All three authors wrote the manuscript

17 


\section{Abstract}

19 Common salvinia, Salvinia minima Baker (Salviniaceae), is a small, floating aquatic fern native to 20 Central and South America that has invaded fresh water bodies in southeastern United States 21 since the 1930s. We examined genetic variation across much of the introduced range of this 22 species in the United States using codominant RAD-seq markers. Data from over 600 variable 23 loci showed a reduction in heterozygosity from east to west in addition to a corresponding 24 trend in assignment of samples to one of two genetic groups. Our data are consistent with 25 previous published work and with the hypothesis that common salvinia had a single 26 introduction on the east end of its current range in the United States. From there it migrated 27 westward, losing genetic diversity during this spread. The data are also consistent with sexual 28 reproduction, although we are unable to estimate the extent of this relative to asexual 29 spreading. Future genetic work should include sampling from the native range to help 30 determine the original sources of North American common salvinia. 


\section{Introduction}

Common salvinia, Salvinia minima Baker (Salviniaceae), is a small, floating aquatic fern native to Central and South America and is believed to be first introduced into wetland habitats in the southeastern United States in the late 1920s (Jacono et al., 2001). According to collection records, S. minima was probably first introduced into widely separated river drainages across the southeastern US starting in the 1930s (Jacono et al., 2001). Salvinia minima has been

cultivated in greenhouse aquaria, backyard ponds, and pools as an ornamental plant since the late 1880s (Weatherby, 1921, 1937; Fernald, 1950). Thus, introduction into natural areas likely occurred due to accidental (i.e. flooding) or intentional release. Since its initial introductions in drainages west of the Florida panhandle, it has colonized rapidly into suitable habitats likely assisted by human movement including recreational watercrafts, but the spread is not as aggressive in the rest of Florida (Jacono et al., 2001). This reduced invasiveness was attributed to the presence of the salvinia weevil, Cyrtobagous salvineae Calder \& Sands which was documented in $68 \%$ of the Florida collections (Jacono et al., 2001). However, there is some question as to whether this is the same species as the $C$. salvineae that is found on the giant salvinia (S. molesta) in Brazil (Calder and Sands, 1985; Madeira et al., 2006; Russell et al., 2017). Salvinia minima can grow and spread rapidly asexually by fragmentation but can also reproduce sexually (De La Sota and Cassá De Pazos, 2001). The base chromosome number in the genus is $\mathrm{n}=$ 9. Salvinia minima is believed to be tetraploid $(2 n=36)$ but hexaploids (possibly hybrid derivatives involving S. minima and S. sprucei Kuhn) have been identified near Manaus, Brazil (De La Sota and Cassá De Pazos, 2001). Two previous genetic studies from more than 10 years ago considered population variation along the Gulf Coast resulting in contrasting findings. An 
57 isozyme study (Hauber and Lingam, unpub) found no variation among populations from Texas to Florida, whereas a study using dominant RAPD markers (Madeira et al., 2003) found considerable within and between population variation across the Southeast. Here we used a DNA sequencing approach that potentially captures thousands of codominant loci to examine

61 genetic variation of introduced populations of S. minima. Our objectives are: 1) To test for multiple introductions of S. minima in thesSoutheastern United States, 2) To examine east west patterns of genetic variation, 3) To determine whether introduced populations are

64 propagating asexually or if within-population variation suggests significant levels of sexual reproduction, and 4) To examine genetic evidence for recent or historical hybridization. We

66 also attempt to explain why different methods detect different levels of genetic variation.

\section{Methods}

69

70 water until DNA extractions. The exceptions were populations 475-481 (Supplementary Table

\subsection{Sampling}

Salvinia minima samples were collected from southeastern (Orange County) Texas east along the US Gulf Coast to central (Marion County) Florida (Fig. 1). Efforts were made to include sites where introductions were reported (Jacono et al., 2001). Salvinia molesta was also collected at four sites, in part, to control for possible adulterated samples of $S$. minima because immature $S$. molesta can be confused with S. minima in the field. Every attempt was made to collect individuals that appeared healthy and separated by $>10 \mathrm{~m}$ from subsequent samples. This was true for both S. minima and S. molesta. Most collections were made fresh and kept in in situ 1), which were collected and stored in silica. Fresh specimens were rinsed in deionized water 
and forceps were used when needed to remove occasional invertebrates, duckweed and biofilm. Approximately $0.1 \mathrm{~g}$ of the healthiest appearing leaflets were excised from the leaf axis for extraction.

\section{2 DNA extraction and Genomic DNA library preparation}

Genomic DNA was extracted using the CTAB method from Neubig et al. (2014). Extractions were assessed for quality and quantity by visualization on a $1 \%$ agarose gel and a NanoDrop instrument (Thermo Scientific, Wilmington, MA). The genomic library was made with a double digestion restriction site-associated DNA sequencing (ddRADseq) protocol (Gompert et al., 2012; Parchman et al., 2012), using EcoR1 and Mse1 to fragment the genomic DNA. Fragments were ligated to barcoded (indexed) oligonucleotides (with barcodes unique to each individual) on the EcoRI ends of the fragments. Samples were then PCR-amplified using iproof high-fidelity DNA polymerase (New England Biolabs) with primers that overlap the ligated oligonucleotides.

All fragments were first mixed with only one other (barcoded) individual, which then were further amplified in duplicate to reduce stochastic variation in PCR amplification, before final pooling of all barcoded samples. The library was then reduced to fragments in the size range of 250-350 bp using a Blue Pippin (Sage Science, Beverly, MA). Quality and quantity were further verified using TapeStation 2200 (Agilent Technologies). The size-selected, multiplexed samples were run on a single lane of Illumina HiSeq 2500 with $100 \mathrm{bp}$ single-end sequencing at Genomic Sequencing and Analysis Facility at the University of Texas at Austin (GSAF).

\section{3 Data processing}


101 Raw Illumina reads were processed with ipyrad v.0.5.15 (Eaton, 2014). This process was carried

102 out twice, with the first round using the entire dataset of 96 samples (including S. molesta

103 samples) to identify low-coverage and failed reads that should be removed from further

104 analyses, as well as to verify replication for quality control. The second round of analysis was

105 performed on a reduced dataset of 63 S. minima individuals and 15 S. molesta, and included a

106 more stringent filtering to remove possible duplicated loci, loci with low coverage, and loci that

107 were not in at least $70 \%$ of samples. All raw DNA sequence data plus every detail of the data

108 processing steps and parameters used are available on Digital Commons

109 (https://doi.org/10.15142/T3VK80). Using ipyrad, samples are first demultiplexed and quality

110 filtered. Within-sample clusters are generated using USEARCH (Edgar, 2010), and reads are

111 aligned using MUSCLE (Edgar, 2004). Error rate and heterozygosity are then estimated, and

112 consensus bases are called and filtered. Finally, clusters were generated across samples, and

113 filters are applied to the resulting data, generating a number of genotype output formats. Due

114 to the lack of a reference genome, ipyrad assembled the data de novo using vsearch (Enns et

115 al., 1990). The clustering threshold was set to $90 \%$ sequence similarity.

117 2. 4 Analyses

118 We confirmed genetic differentiation of S. minima from S. molesta using a neighbor-joining (NJ)

119 tree using adegenet (Jombart, 2008). Because introduced species often have reduced genetic

120 variation, we next used two different methods to identify natural genetic clusters. If both

121 methods converge, we can have more confidence in our inferences. These methods used are

122 Discriminant Analysis of Principal Components (DAPC; Jombart and Collins, 2015) using 
adegenet 2.0.1 in R, and STRUCTURE 2.3.4 (Pritchard et al., 2000; Falush et al., 2003; Falush et al., 2007; Hubisz et al., 2009). In both analyses, individuals were treated independently from each other, without any predefined associations due to geographic proximity. DAPC is a multivariate analysis that builds on the strengths of Principal Component Analysis (PCA) and Discriminant Analysis (DA), where genetic structure among individuals is determined such that within-group variation is minimized while between-group variation is maximized. A k-means algorithm is also used to infer genetic clusters and a statistical measure of goodness of fit using Bayesian Information Criterion (BIC) taken across the number of clusters to infer the optimal number of clusters and individual assignment to each cluster. STRUCTURE uses a Bayesian clustering approach that first randomly assigns individuals to pre-determined groups (K groups): S. minima was tested against 2 through 6 groups, while $S$. molesta was tested across 1 through 4 groups. A Markov Chain Monte Carlo (MCMC) estimation is applied wherein individuals are re-assigned to each group based upon variant frequency estimates. In our analyses, we used a burn-in of 500,000 followed by $1,000,000$ iterations with 50 replicates for each of the tested $\mathrm{K}$ groups. For each $\mathrm{K}$ group tested, a population Q-matrix is formed for each of the 50 replicates, which shows the average individual membership coefficient to each cluster. To determine the "optimal" $\mathrm{K}$ value from the STRUCTURE output, we implemented STRUCTURE HARVESTER (Earl and vonHoldt, 2012). This web-based program processes STRUCTURE results across all tested $\mathrm{K}$ groups, and performs the Evanno method (Evanno et al., 2005) for detecting the optimal number of $\mathrm{K}$ groups that best fit the dataset. Note that the Evanno method does not always select the optimal $\mathrm{K}$ groups, most notably if the best group is $\mathrm{K}=1$. Therefore, we also assessed the effect of number groups (K) 
145 using a graphical representation resulting from CLUMPAK (Kopelman et al., 2015), which

146 implemnts STRUCTURE HARVESTER (Earl and vonHoldt, 2012), CLUMPP (Jakobsson and

147 Rosenberg, 2007), and DISTRUCT (Rosenberg, 2004). Detailed output is shown in our data

148 analyses on Digital Commons (https://doi.org/10.15142/T3VK80). Observing the STRUCTURE

149 patterns as K groups increases, one can assess whether adding each group provides additional,

150 meaningful structure to the data rather than mirroring the structure that is already there. After

151 selecting the optimal K group, we visualized both the DAPC and STRUCTURE results using a

152 custom python script.

We further examined the relationship of longitude with both cluster assignment and

154 heterozygosity using linear regression. Levels of genetic variation were explored by examining

155 patterns of heterozygosity across loci and individuals. Details for all analyses are available on

156 Digital Commons (https://doi.org/10.15142/T3VK80)

158 3. Results

159 Initial analyses with both S. molesta and S. minima samples were quality-filtered with very low 160 stringency to maintain as many loci as possible that are shared across the two species. Eight 161 samples were considered failures due to extremely low sample coverage. Unfortunately, most 162 of these were from herbarium specimens from the native range of S. minima. After filtering, we 163 retained 88 samples with 21,059 loci. Distinct genetic differentiation between S. minima and S. 164 molesta was confirmed using the NJ tree (not shown). Collection population 461 included 9 165 individuals of S. molesta, and a single of S. minima, confirmed by both the NJ tree and DAPC 166 cluster assignment. Individual 465_2 was a far outlier as observed in a scatter plot of the DAPC 
167 results. Subsequent analyses focused on S. minima, with select comparisons to S. molesta, but 168 always treating the species separate and with their own loci.

After data quality filtering the S. minima dataset, we retained 687 SNP loci across 63

170 individuals. DAPC results showed two genetic groups across S. minima with $100 \%$, or nearly so,

171 assignment to one group or the other (Fig. 2). From STRUCTURE, plots of the optimal

172 alignment(s) from CLUMPP for each K group clearly show that there is either a single genetic

173 cluster, or two. Where $\mathrm{K}=2$, CLUMPP resulted in two main alignments in which 23 of the 50

174 STRUCTURE replicates resulted in two distinctly separated genetic groups which were similar to

175 that observed in DAPC. Whereas DAPC assigned individuals exclusively, or nearly so, to one

176 cluster or the other, STRUCTURE revealed samples with lower individual assignment to each of

177 the two clusters. However, the majority assignment in STRUCTURE was to the same cluster as in

178 DAPC (Fig. 2). This probability cluster assignment from STRUCTURE/CLUMPP was used to

179 further examine their cluster relationship with geography. The proportion of individuals

180 assigned to cluster 1 was regressed against the longitude positions. We detected a positive 181 relationship with a slope of 0.04218 , an intercept of $4.21, r=0.3895$, and $p=0.0016$ (Fig. 3), 182 indicating that a null hypothesis of no relationship should probably be rejected. Furthermore, 183 we estimated heterozygosity for S. minima, across loci (mean 0.153; S.E. 0.006; Fig. 4) and 184 across individuals (mean 0.144; S.E. 0.022; Fig. 5). The general patterns are consistent with 185 typical distributions for neutral loci (Nei et al., 1976). We also performed a linear regression 186 analysis of heterozygosity (of individuals) on longitude (Fig 6). The relationship was significant, 187 with a slope of 0.002 , intercept of $0.334, r=0.258$, and $p=0.041$. This provides some evidence 188 that heterozygosity is higher in the eastern end of the range. We do not have data from native 
range, but the levels of heterozygosity detected here are consistent with sexual reproduction in the introduced range of S. minima. If populations were spreading only via asexual cloning, then heterozygosity would remain the same. With asexual reproduction of related individuals, heterozygosity is expected to drop each generation. Thus, the east-to-west drop in heterozygosity is consistent with sexual reproduction at some point since introduction.

We also provide a neighbor-joining tree of individuals based on genetic distance (Figure 7) depicting relationships among samples in this study.

The S. molesta dataset was filtered to 15 individuals and 461 loci. Both DAPC and STRUCTURE resulted in a single genetic cluster. Estimated heterozygosity across loci for $S$. molesta (mean 0.1669; S.E. 0.0078) was similar to that observed in S. minima.

\section{Discussion}

Our analysis of over 600 co-dominant genetic markers revealed more variation in Salvinia minima than in a previous (unpublished) isozyme study. Our results are consistent with the patterns of variation detected using dominant RAPD markers (Madeira et al., 2003). The two analyses (DAPC and STRUCTURE) we used to examine genetic structure indicated two genetic clusters and it is possible that these represent separate introductions. However, it is probably more likely that the pattern is caused by introduction in the eastern end of the range with loss of variation during westward migration. This hypothesis is also supported by the reduction in heterozygosity in the west end of the range of S. minima.

Because we have no strong evidence for more than one introduction, we cannot fully address our initial goal of determining if there has been hybridization between diverse 
211 introduced populations. During some plant invasions, multuple introductions can result in an

212 increase in genotypic variation by hybridization and recombination between invasive genotypes

213 (Lavergne and Molofsky, 2007). Such hybridization can overcome the effects of genetic

214 bottlenecks associated with invasions. We detected no such patterns for S. minima.

215 Depicting relationships among individuals based on variation at polymorphic markers is

216 inherently challenging. Tree figures and even 2- and 3-dimentional PCA depictions can miss

217 underlying genetic structure within genomes. These problems are exacerbated when

218 comparing samples that are in the introduced range of a species in which levels of variation are

219 likely reduced. Our approach, involving large numbers of codominant markers, was aimed to

220 increase the chances of detecting multiple origins, and we find no strong evidence for more

221 than a single origin of S. minima in the regions where we sampled.

222

We suspect that the lack of variation in the isozyme study is a function of reduced

223 variation at the protein level compared to DNA in general (Kreitman, 1983; Casillas and

224 Barbadilla, 2017) and because isozymes target important metabolic genes, whereas RAPD and

225 ddRAD-seq use nonspecific genomic regions.

We detected moderate levels of heterozygosity and nucleotide variation that are

227 consistent with some sexual reproduction. Furthermore, variation in heterozygosity across

228 individuals provides further evidence that sexual reproduction is occurring in the introduced

229 range. This contrasts with the assumption that S. minima is sterile and spreads only via clonal

230 reproduction (Jacono et al., 2001; Morgan, 2009). Still, sexual reproduction may indeed be rare,

231 and, if we are examining the descendants of a single introduction then even sexual

232 reproduction is not going to generate much additional variation, only new combinations of 
233 standing genetic variation. We did not perform genotype frequency analysis because sample

234 sizes are small and because failure to fit random mating patterns of genotypes can be

235 confounded in populations of an introduced species (where matings would likely be among

236 relatives) and a plant that spreads clonally. Some of the interesting questions that have

237 emerged from this study are: 1) Is there more support for sexual propagation and, if so, how

238 common is it? 2) How is it that, except for the east to west migration, there does not appear to

239 be any evidence of distribution of genetic variation by distance? 3) How does variation in the

240 native range compare with that in the introduced populations? Selecting a few populations

241 from both the western and eastern ends of the introduced range, and collecting larger samples

242 at these sites would allow for a genotype frequency analysis to test for sexual reproduction and

243 better assess population genetic structure. Future work on common salvinia in the native range

244 and comparison with the complete introduced range populations would enable inference as to

245 the origin of introduced populations. Samples we obtained from the native range unfortunately

246 exhibited low sample coverage and could not be included in the analysis. This might be due to

247 poor preservation of herbarium specimens. Aquatic species require special attention to higher

248 than normal silica to specimen ratio. Extracting DNA from fresh specimens collected across the

249 native range of South America would be a more reliable strategy. Here we find that S. minima

250 had a single origin in southeastern part of its introduced range in USA, and subsequently moved

251 westward. We find evidence of sexual reproduction and maintenance of moderate levels of

252 genetic variation.

253

254 Acknowledgments: 
255 Thanks to Zach Gompert, Aaron Duffy, and Deepthi Lingam for assistance with this project. We

256 thank the Rev. J. H. Mullahy Endowment for funding this research, and Kathlene Pryer, Alan

257 Smith, Colette Jacono, and Larry Raymond for access to herbarium specimens

258

259 


\section{References}

261 Calder, A.A., Sands, D.P.A., 1985. A new Brazilian Cyrtobagous Hustache (Coleoptera: Curculionidae) introduced into Australia to control salvinia. Journal of the Australian Entomological Society 24,

264 Casillas, S., Barbadilla, A., 2017. Molecular population genetics. Genetics 205, 1003-1035.

265 De La Sota, E.R., Cassá De Pazos, L.A., 2001. Two cytotypes and a new hybrid in Salvinia Séguier. Acta 266 Amazonica 31, 557-557.

267 Earl, D.A., vonHoldt, B.M., 2012. STRUCTURE HARVESTER: a website and program for visualizing STRUCTURE output and implementing the Evanno method. Conservation Genetics Resources 4,

Eaton, D.A., 2014. PyRAD: assembly of de novo RADseq loci for phylogenetic analyses. Bioinformatics 30, 1844-1849.

Edgar, R.C., 2004. MUSCLE: multiple sequence alignment with high accuracy and high throughput. Nucleic Acids Research 32, 1792-1797.

Edgar, R.C., 2010. Search and clustering orders of magnitude faster than BLAST. Bioinformatics 26, 24602461.

Falush, D., Stephens, M., Pritchard, J.K., 2003. Inference of population structure using multilocus genotype data: Linked loci and correlated allele frequencies. Genetics 164, 1567-1587.

Falush, D., Stephens, M., Pritchard, J.K., 2007. Inference of population structure using multilocus genotype data: Dominant markers and null alleles. Molecular Ecology Notes 7, 574-578. 
Fernald, M.L., 1950, Gray's Manual of Botany: a handbook of the flowering plants and ferns of the central and northeastern United States and adjacent Canada. American Book Company, New York.

Gompert, Z., Lucas, L.K., Nice, C.C., Fordyce, J.A., Forister, M.L., Buerkle, C.A., 2012. Genomic regions with a history of divergent selection affect fitness of hybrids between two butterfly species. Evolution 66, 2167-2181.

Hubisz, M.J., Falush, D., Stephens, M., Pritchard, J.K., 2009. Inferring weak population structure with the assistance of sample group information. Molecular Ecology Resources 9, 1322-1332.

Jacono, C.C., Davern, T.R., Center, T.D., 2001. The adventive status of Salvinia minima and S. molesta in the southern United States and the related distribution of the weevil Cyrtobagous salviniae. Castanea 66, 214-226.

Jakobsson, M., Rosenberg, N.A., 2007. CLUMPP: a cluster matching and permutation program for dealing with label switching and multimodality in analysis of population structure. Bioinformatics 23, 1801-1806.

Jombart, T., 2008. adegenet: a R package for the multivariate analysis of genetic markers. Bioinformatics 24, 1403-1405.

Jombart, T., Collins, C., 2015, A tutorial for discriminant analysis of principle components (dapc) using adegenet 2.0.0.

Kopelman, N.M., Mayzel, J., Jakobsson, M., Rosenberg, N.A., Mayrose, I., 2015. CLUMPAK: a program for identifying clustering modes and packaging population structure inferences across K. Molecular Ecology Resources 15, 1179-1191.

Kreitman, M., 1983. Nucleotide polymorphism at the alcohol dehydrogenase locus of Drosophila melanogaster. Nature 304, 412-417. 
Lavergne, S., Molofsky, J., 2007. Increased genetic variation and evolutionary potential drive the success of an invasive grass. Proceedings of the National Academy of Sciences (USA) 104, 3883-3888.

Madeira, P.T., Jacono, C.C., Tipping, P., Van, T.K., Center, T.D., 2003. A genetic survey of Salvinia minima in the southern United States. Aquatic Botany 76, 127-139.

Madeira, P.T., Tipping, P.W., Gandolfo, D.E., Center, T.D., Van, T.K., O'brien, C.W., 2006. Molecular and morphological examination of Cyrtobagous sp. collected from Argentina, Paraguay, Brazil, Australia, and Florida. BioContol 51, 679-701.

Morgan, V.H., 2009, Salvinia minima. USGS: Nonindigenous Aquatic Species Database. Gainesville, FL. . USGS: Nonindigenous Aquatic Species Database, Gainesville, FL.

Nei, M., Fuerst, P.A., Chakraborty, R., 1976. Testing the neutral mutation hypothesis by distribution of single locus heterozygosity. Nature 262, 491.

Neubig, K.M., Whitten, W.M., Abbott, J.R., Elliott, S., Soltis, D.E., Soltis, P.S., 2014, Variables Affecting DNA Preservation in Archival DNA Specimens. In: Applequist, W.L., Campbell, L.M. (Eds.), DNA Banking for the 21st Century: Proceedings of the U.S. Workshop on DNA Banking. The William L. Brown Center at the Missouri Botanical Garden, St. Louis, MO, USA.

Parchman, T.L., Gompert, Z., Mudge, J., Schilkey, F.D., Benkman, C.W., Buerkle, C.A., 2012. Genomewide association genetics of an adaptive trait in lodgepole pine. Molecular Ecology 21, 29913005.

Pritchard, J.K., Stephens, M., Donnelly, P., 2000. Inference of population structure using multilocus genotype data. Genetics 155, 945-959.

Rosenberg, N.A., 2004. DISTRUCT: a program for the graphical display of population structure. Molecular Ecology 4, 137-138. 
329 Russell, A., McKay, F., Moshman, L., Madeira, P.T., Diaz, R., Johnson, S., Cibils, X., Blair, Z., 2017. Surveys in Argentina and Uruguay reveal Cyrtobagous salviniae (Coleoptera: Curculionidae) populations adapted to survive temperate climates in southeastern USA. v. 107.

332 Weatherby, C.A., 1921. Other records of Salvinia natans in the United States. American Fern Journal 11, 50-53. 


\section{Figure legends}

Figure 1. Map of sampling locations for Salvinia minima and S. molesta in southeastern United States. Colours denote STRUCTURE assignment at K=3: green = cluster 1; blue = cluster 2; magenta $=$ cluster $3($ S. molesta) .

Figure 2. Results of Structure analysis (top panel) and DAPC (bottom panel) of S. minima, for two genetic clusters $(K=2)$. Green $=$ cluster 1 ; blue $=$ cluster 2.

Figure 3. Scatter plot of proportion of sample clustering with cluster 1 as a function of longitude

Figure 4. The distribution of mean heterozygosity across loci for S. minima.

Figure 5. The distribution of mean heterozygosity across individuals for S. minima.

Figure 6. Linear regression analysis of heterozygosity (of individuals) on longitude.

Figure 7. Neighbor-joining tree of individuals of S. minima. Colours denote genetic assignment based on STRUCTURE at $\mathrm{K}=2$. Green = cluster 1 ; blue = cluster 2. 


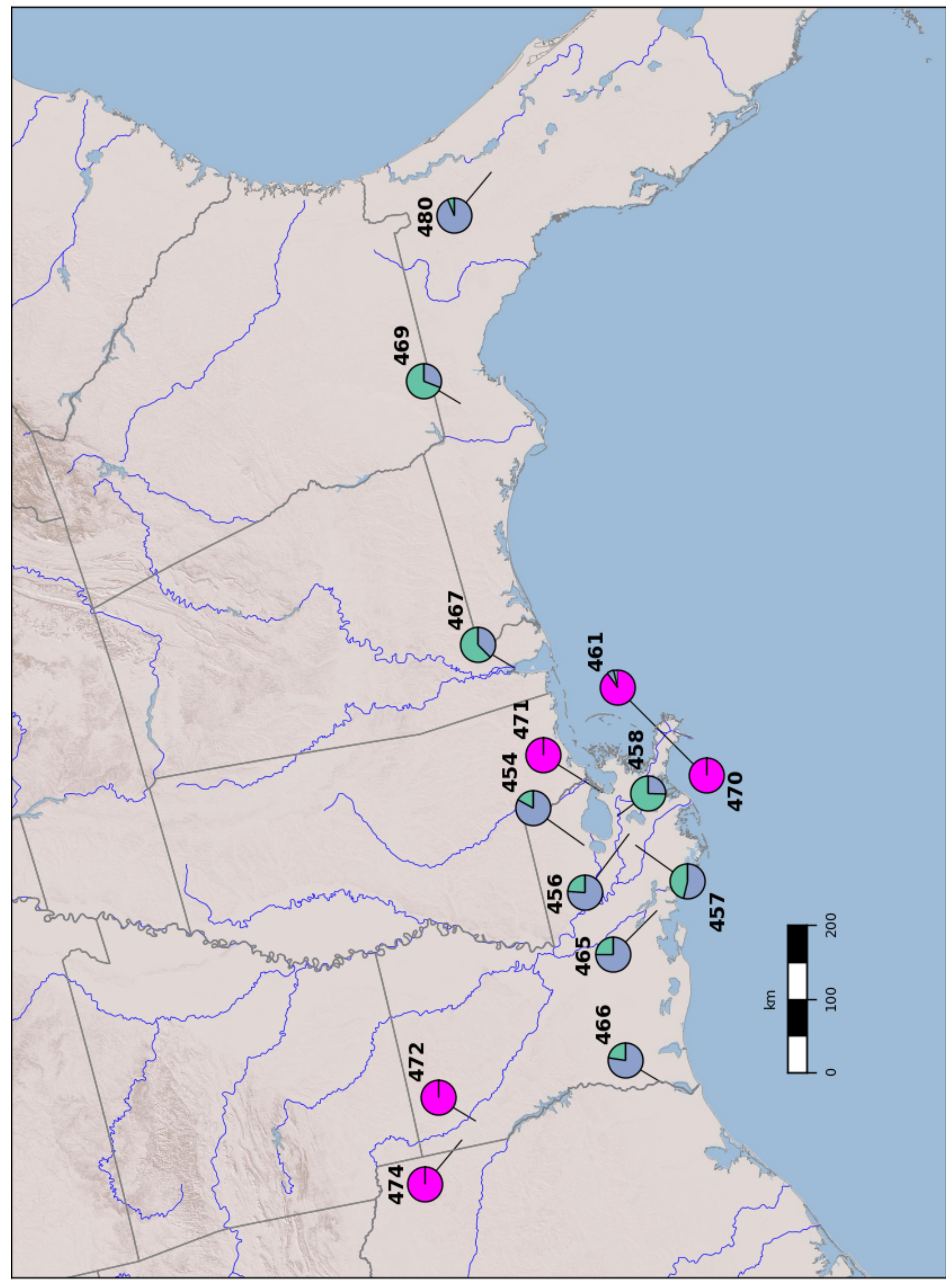




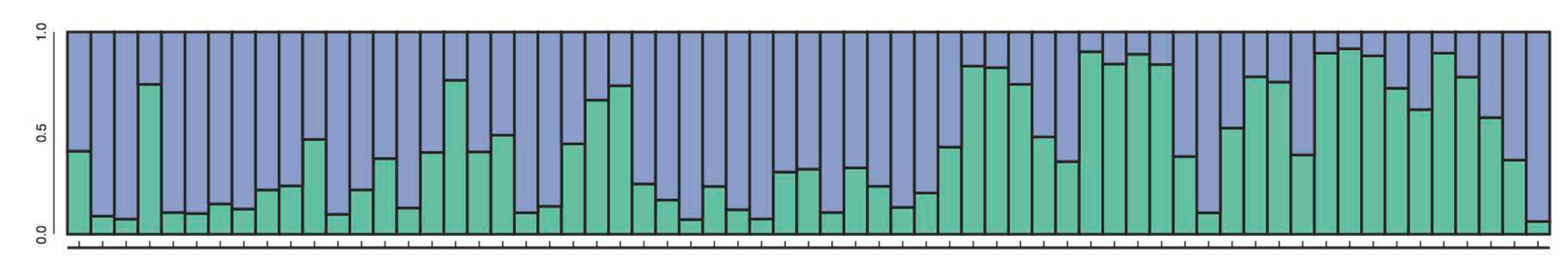



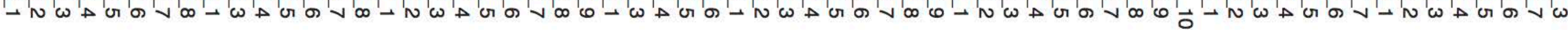

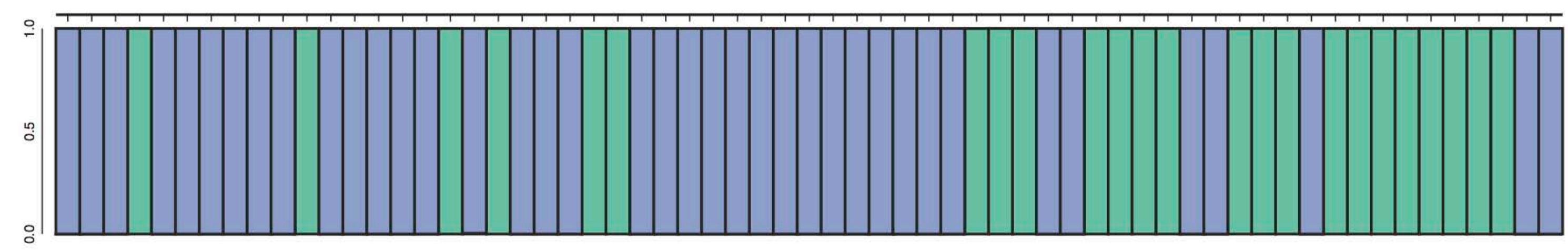




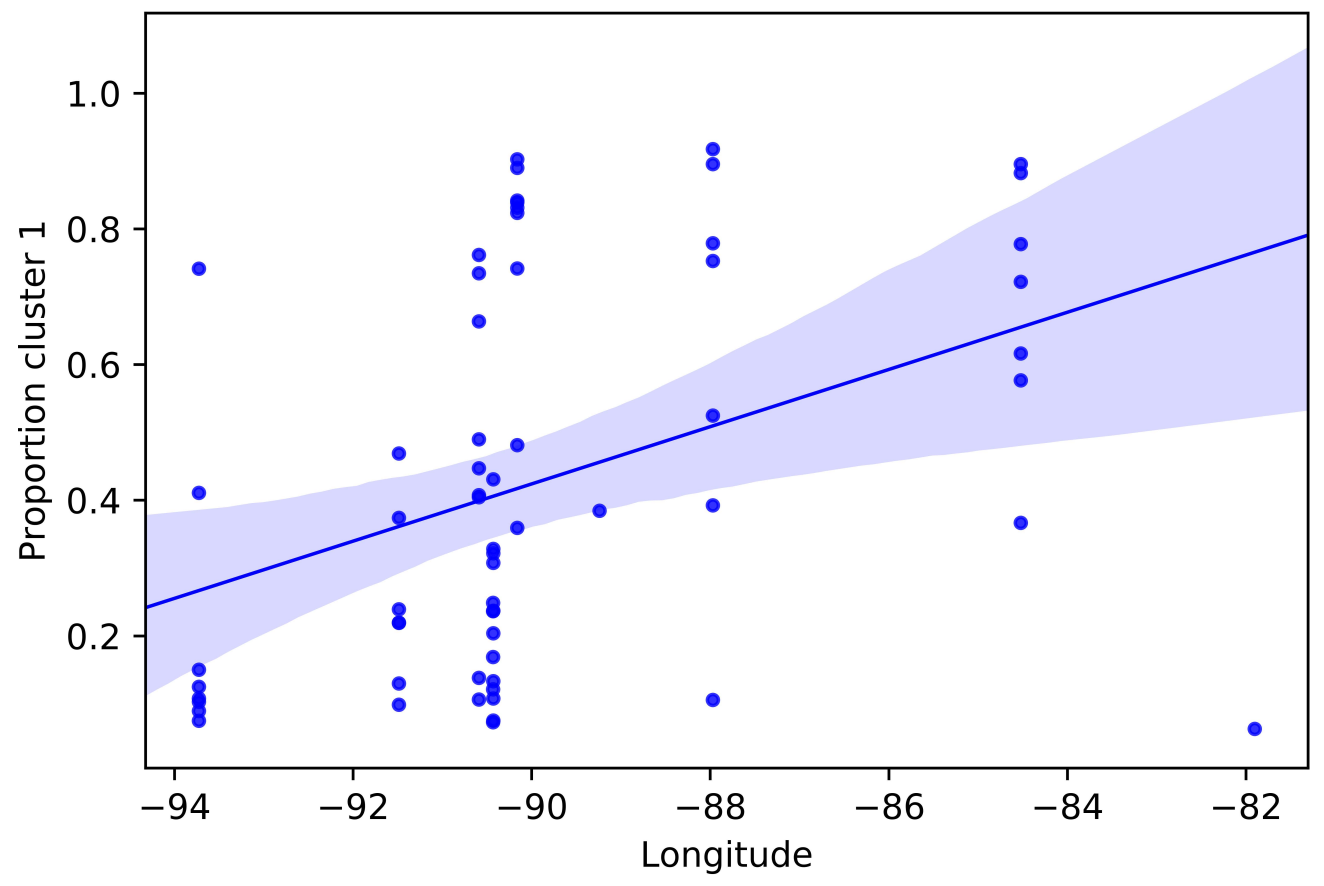




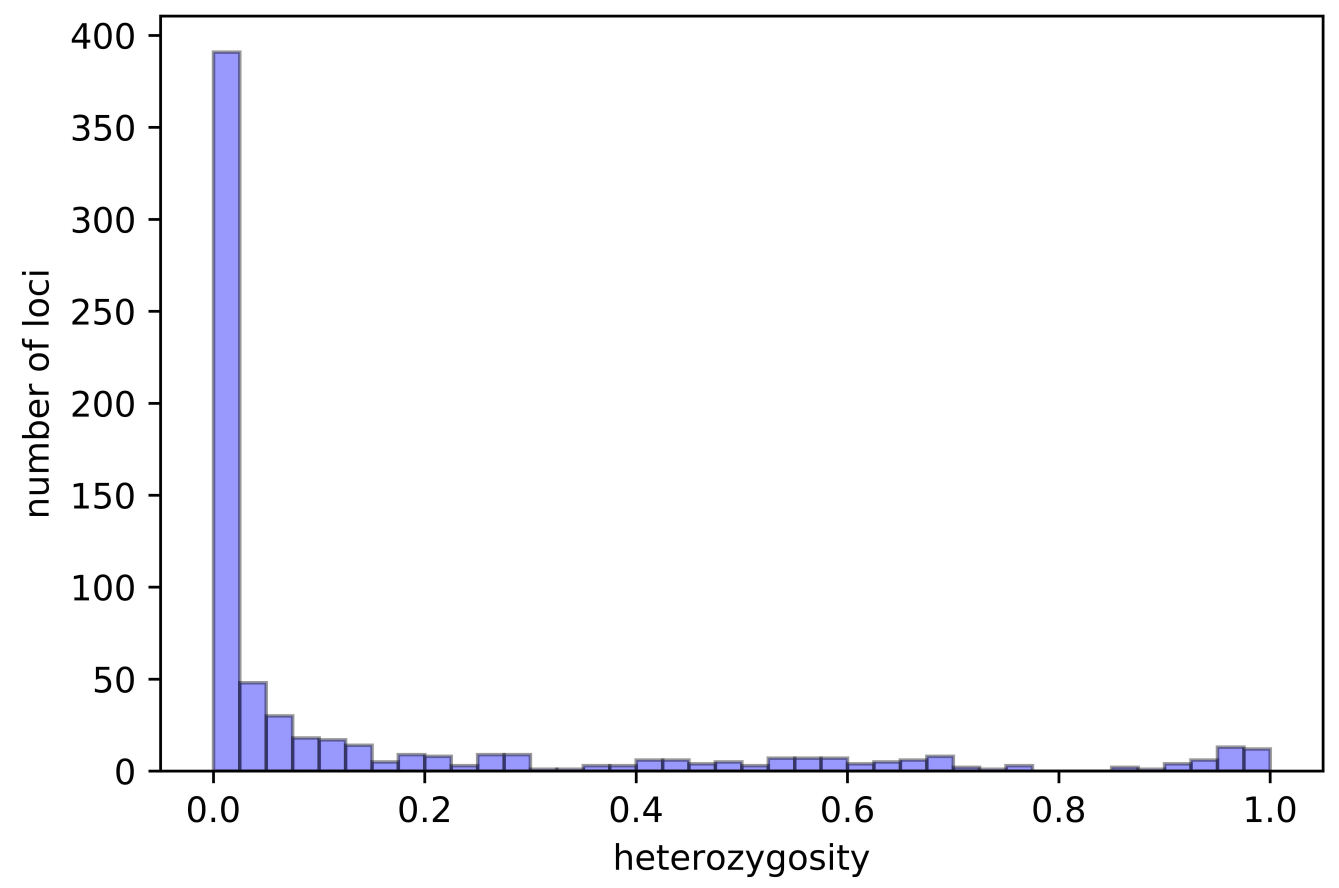




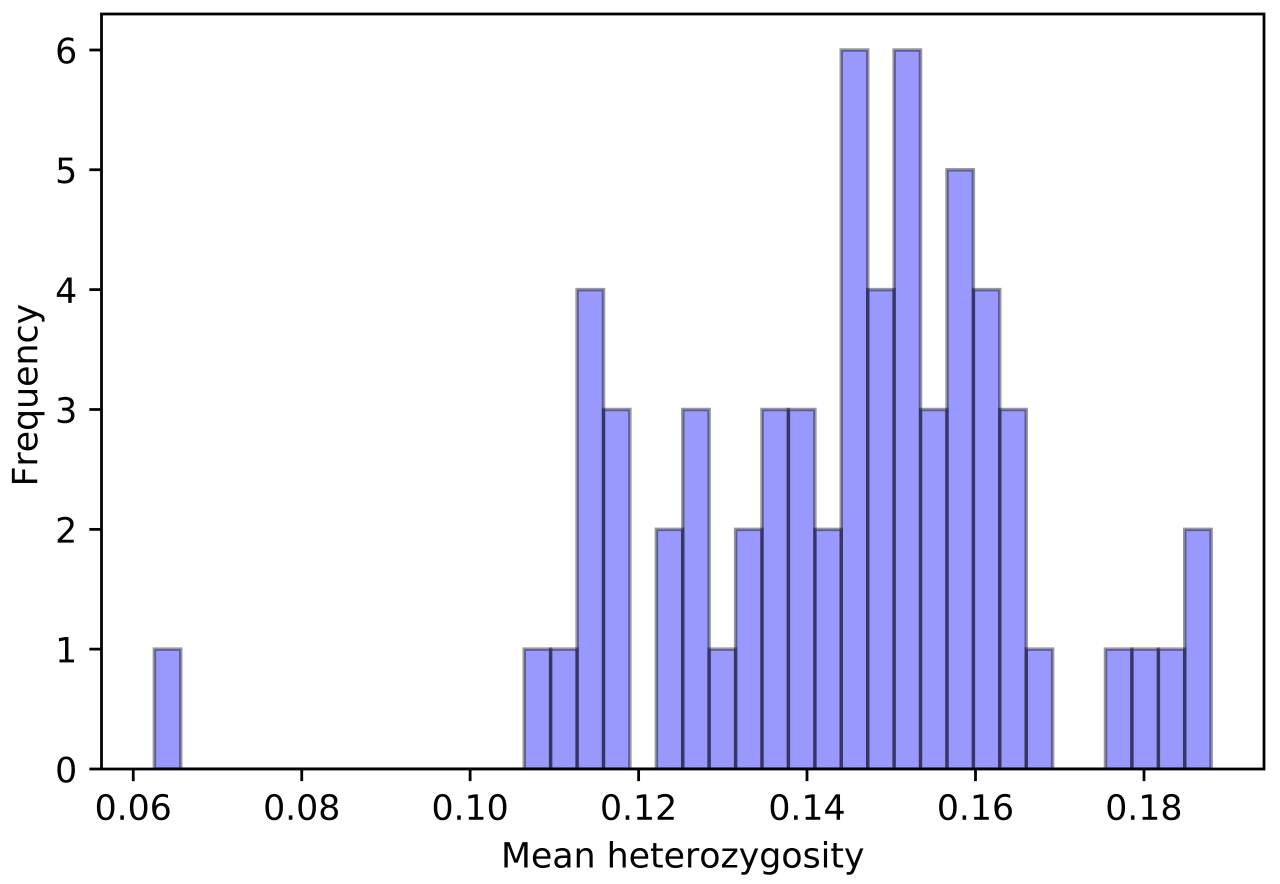




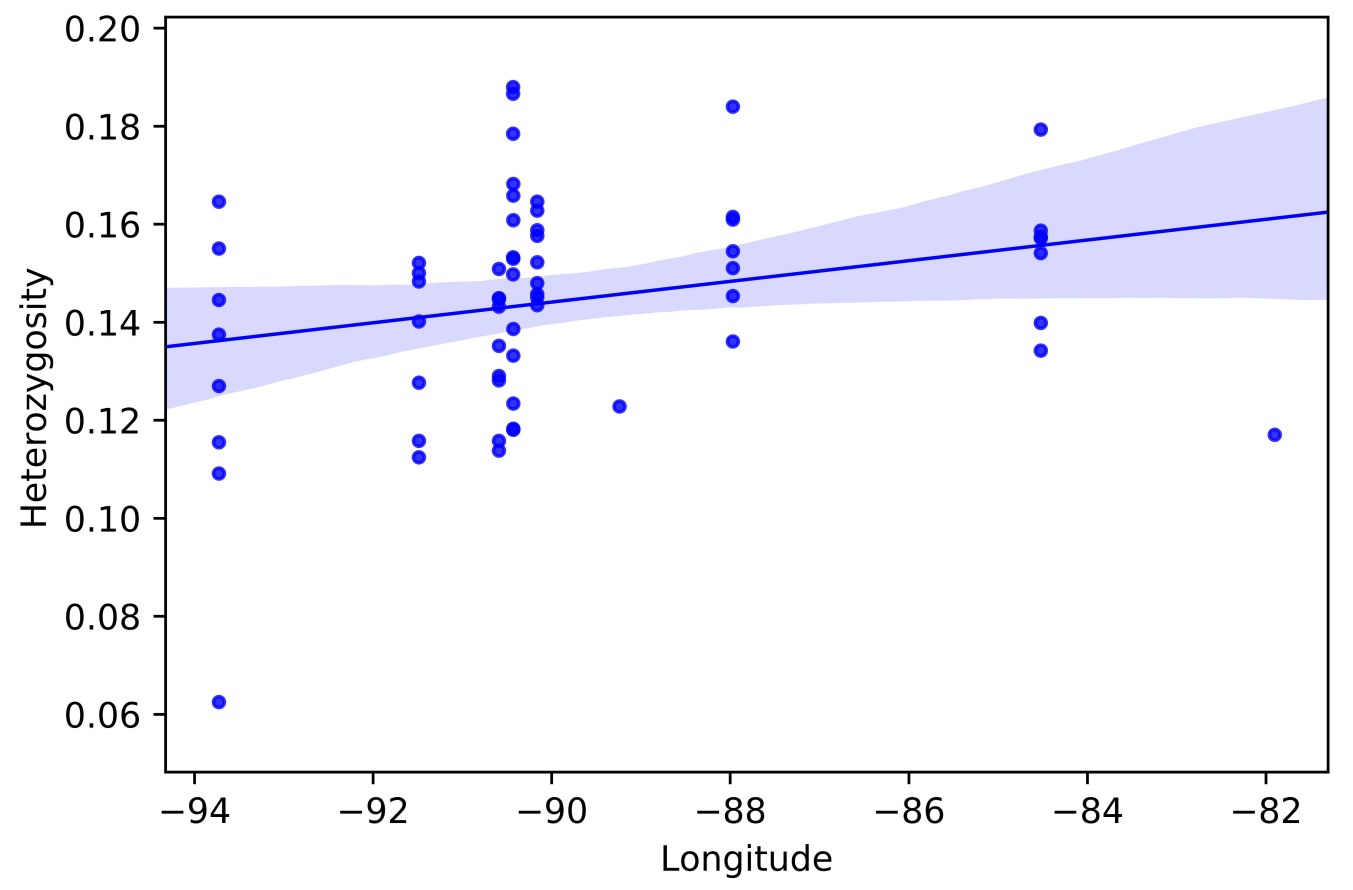




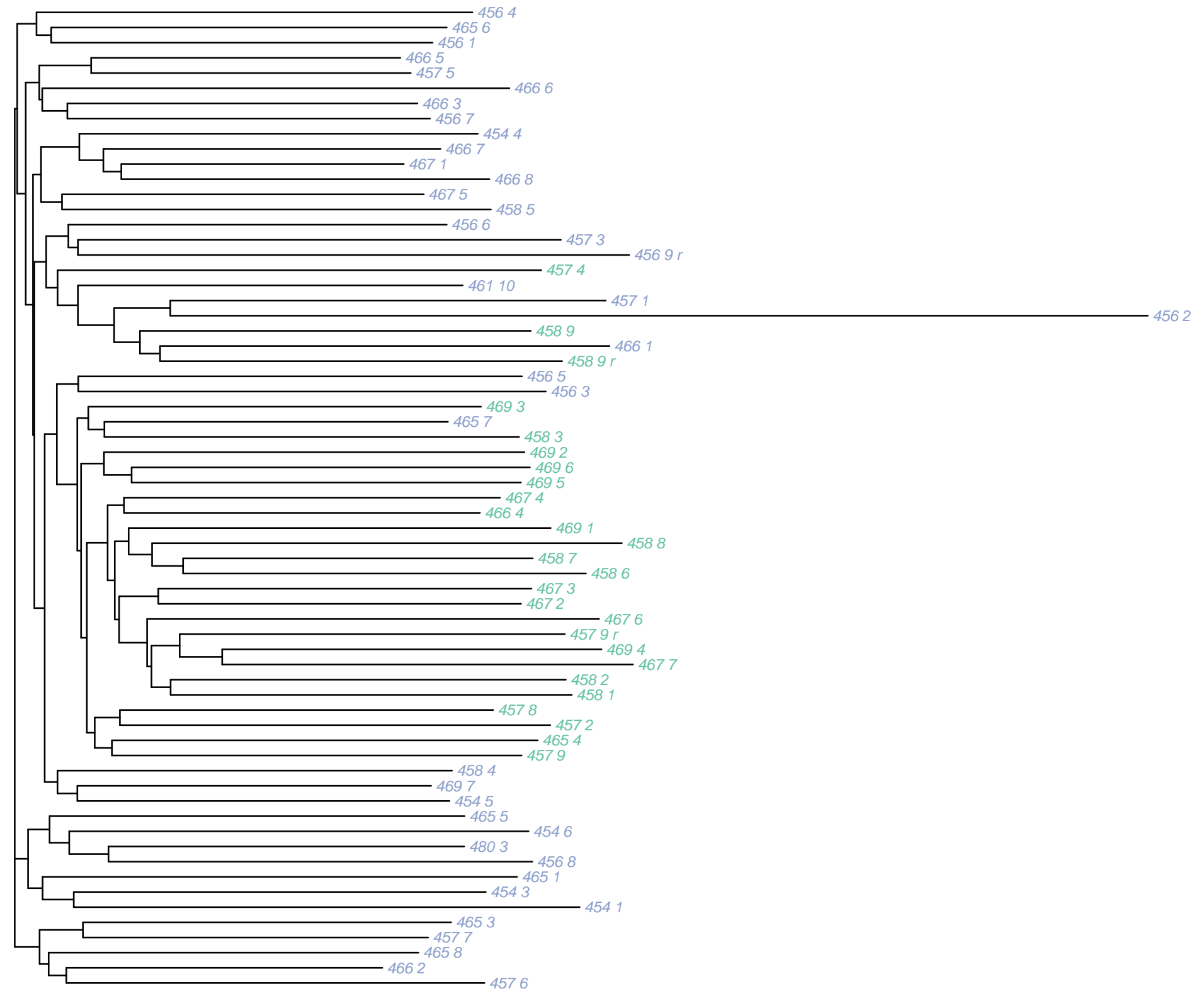


Supplementary Table 1. Locality data for samples of Salvinia collected

\begin{tabular}{|c|c|c|c|c|c|c|c|c|}
\hline No. & Species & Vicinity or collection & MGRS & Longitude & Latitude & County & State & Country \\
\hline 454 & S. minima & Lafitte & 15RYP4673966692 & -90.43161 & 30.40705 & Tangipahoa Parish & Louisiana & USA \\
\hline 456 & S. minima & Paradis & 15RYP4829508439 & -90.42908 & 29.88157 & Saint Charles Parish & Louisiana & USA \\
\hline 457 & S. minima & Kraemer & 15RYP3275602547 & -90.59113 & 29.83148 & Lafourche Parish & Louisiana & USA \\
\hline 458 & S. minima & Jefferson & 15RYP7384217364 & -90.16254 & 29.9566 & Jefferson Parish & Louisiana & USA \\
\hline 461 & S. molesta & S. of Romere Pass & 16RBT8225638232 & -89.24077 & 29.25421 & Plaquemines Parish & Louisiana & USA \\
\hline 465 & S. minima & Franklin & 15RXN4623093775 & -91.48747 & 29.76564 & Saint Mary Parish & Louisiana & USA \\
\hline 466 & S. minima & Orange & 15RVP2994231386 & -93.72719 & 30.1117 & Orange County & Texas & USA \\
\hline 467 & S. minima & Mobile & 16RDU0699094023 & -87.97098 & 30.67533 & Baldwin County & Alabama & USA \\
\hline 469 & S. minima & Lake Tallequin & 16RGU3799671081 & -84.52154 & 30.44838 & Leon County & Florida & USA \\
\hline 470 & S. molesta & S. of Romere Pass & 16RBT8225638232 & -89.24077 & 29.25421 & Plaquemines Parish & Louisiana & USA \\
\hline 471 & S. molesta & Venetian Isles & 16RBU2871329527 & -89.81411 & 30.0668 & Orleans Parish & Louisiana & USA \\
\hline 472 & S. molesta & Wallace Lake & 15SVR3293576352 & -93.71251 & 32.32201 & Caddo Parish & Louisiana & USA \\
\hline 474 & S. molesta & Cross Lake & 15SVR1243998972 & -93.93234 & 32.52463 & Caddo Parish & Louisiana & USA \\
\hline 475 & S. minima & Pryer 5291 & 16PFS8179843964 & -85.33963 & 10.3444 & & Guanacaste & Costa Rica \\
\hline 476 & S. minima & Cult. Pryer 2364 & & & & & Buenos Aires & Argentina \\
\hline
\end{tabular}




\begin{tabular}{|c|c|c|c|c|c|c|c|c|}
\hline 477 & S. minima & Parana, Brazil; & Cordeiro \& Pereira 1531 & & & & & Brazil \\
\hline 478 & S. minima & Sanders 9743 & 16QBF5431498765 & -89.33333 & 18.96666 & & Quintana Roo & Mexico \\
\hline 479 & S. minima & TJ Killeen 6824 & 20LNJ6663867789 & -62.38084 & -14.76306 & & Santa Cruz & Bolivia \\
\hline 480 & S. minima & Jacono 88924 & 17RMN1248149838 & -81.90181 & 29.3747 & Marion County & Florida & USA \\
\hline 481 & S. minima & Jacono 890 & 17RLN6000087613 & -82.44731 & 29.71077 & Alachua County & Florida & USA \\
\hline
\end{tabular}

